\title{
Introducing the Epidemiology Council of the International Society for Physical Activity and Health
}

\author{
Brigid M. Lynch, Andrea Ramirez Varela, and Terry Boyle
}

Since the 1990s, a range of new statistical approaches has been developed to improve causal inference in observational research. The last 2 decades has also seen the development of more sophisticated methods to quantitatively estimate the impact of measurement error, exposure misclassification, and selection bias on the results of observational studies. Physical activity epidemiology has not adopted these methodological advances as quickly as other disciplines.

To address this, the Epidemiology Council of the International Society for Physical Activity and Health (ISPAH) was recently launched. The aim of the Epidemiology Council is to raise the profile of newer epidemiological methods that can help improve causal inference in physical activity research. In our article Approaches to Improve Causal Inference in Physical Activity Epidemiology, ${ }^{1}$ published in this issue of the Journal of Physical Activity and Health, we provide a brief overview of some contemporary methods that we believe may be helpful for physical activity researchers to understand and begin applying within their own work.

The Epidemiology Council will provide opportunities for physical activity researchers wishing to upskill. For example, the Council will provide members with the following:

- Newsletters describing key concepts and methods and highlighting recent publications that have employed causal inference or quantitative bias analysis methods to address physical activity-related questions.

- A virtual journal club, where members can contribute to discussion and debate about causal inference methods via Twitter. Members will be provided with a copy of a scientific article 2 weeks prior to the event and some initial discussion points. A date and time for the Twitter journal club (with unique hashtag) will also be supplied.

- Pre-ISPAH or post-ISPAH Congress workshops that help participants work through an application of analytic method. For example, we may run a workshop on the parametric g-formula, where we would deliver a lecture about the method, provide an example paper for participants to review, and then work through the Stata code (Stata Corp, College Station, TX) on running this example analysis.
We would also like to take this opportunity to announce the flagship project of the Epidemiology Council's first term: the Physical Activity Cohort Study Repository. We plan to conduct an extensive review of existing cohort studies worldwide, to establish which have collected data on physical activity and sedentary behavior (self-report and/or device-based measurement). We will compile a central repository of information about these cohorts, including details such as size, location, years of baseline recruitment, and broad demographics of the cohort; how and when physical activity and/or sedentary behavior were measured; links to cohort profiles or other publications outlining the details of the study; and links to study websites with information about the study and how to request access to the data (if applicable). This resource will encourage researchers to return to existing cohort studies with multiple assessment time points and apply contemporary causal inference methods to provide more robust evidence, thus helping to maximize investment into cohort studies. The Physical Activity Cohort Study Repository will also identify opportunities for international collaboration, including forging research links that will help build capacity in low- and middleincome countries.

The Physical Activity Cohort Study Repository project will be recruiting ISPAH members to assist with the review in the near future. Expressions of interest can be directed to Dr A.R.V. (aravamd@gmail.com).

\section{Acknowledgment}

B.M.L. is supported by a Mid-Career Research Fellowship from the Victorian Cancer Agency (MCRF 18005).

\section{Reference}

1. Lynch BM, Dixon-Suen SC, Ramirez Varela A, et al. Approaches to improve causal inference in physical activity epidemiology. J Phys Act Health; 17(1):80-84. doi:10.1123/jpah.2019-0515 